\title{
Bloodlines: mammals, leeches, and conservation in southern Asia
}

\author{
MICHAEL TESSLER ${ }^{1,2,3}$, SARAH R. WEISKOPF ${ }^{4}$, LILY BERNIKER $^{3}$, REBECCA \\ HERSCH $^{2}$, KYLE P. McCARTHY ${ }^{4}$, DOUGLAS W. YU ${ }^{5,6} \&$ MARK E. SIDDALL ${ }^{1,2,3}$
}

${ }^{1}$ Richard Gilder Graduate School, American Museum of Natural History, Central Park West at 79th Street, New York, NY 10024, USA

${ }^{2}$ Sackler Institute for Comparative Genomics, American Museum of Natural History, Central Park West at 79th Street, New York, NY 10024, USA

${ }^{3}$ Division of Invertebrate Zoology, American Museum of Natural History, Central Park West at 79th Street, New York, NY 10024, USA

${ }^{4}$ Department of Entomology and Wildlife Ecology, University of Delaware, 531 South College Avenue, Newark, DE 19716, USA

${ }^{5}$ State Key Laboratory of Genetic Resources and Evolution, Kunming Institute of Zoology, 32 Jiaochang Dong Lu, Kunming, Yunnan 650223, China

${ }^{6}$ School of Biological Sciences, University of East Anglia, Norwich Research Park, Norwich, Norfolk NR4 7TJ UK

\author{
Running title: Mammals, leeches, and conservation \\ Correspondence to: Michael Tessler. E-mail: mtessler@amnh.org \\ Southern Asia is a biodiversity hotspot both for terrestrial mammals and for leeches. Many \\ small-mammal groups are under-studied in this region, while other mammals are of known
}


conservation concern. In addition to standard methods for surveying mammals, it has recently been demonstrated that residual bloodmeals within leeches can be sequenced to find mammals in a given area. While these invertebrate-parasite-derived DNA (iDNA) methods are promising, most of the leech species utilized for this type of survey remain unevaluated, notwithstanding that their diversity varies substantially. Here we examine approximately 750 individual leech specimens in the genus Haemadipsa across a large range in southern Asia (Bangladesh, Cambodia, and China), specifically reviewing the diversity of mammals they feed on and their own genetic structuring. Leeches were found to feed on a considerable variety of mammals, corroborating prior studies. Additionally, leeches were found to have fed both on bats and on birds, neither of which has previously been recorded with this method. The genetic structuring of the leeches themselves revealed 15 distinct clades of which only two corresponded to previouslycharacterized species, indicating that much work is needed to finalize classifications in this genus. Most importantly, with regards to mammal conservation, leeches in these clades appear to feed on a broad range of mammals.

Key words: biodiversity, bloodmeal, conservation, Haemadipsa, iDNA, leech, mammal, survey 


\section{Introduction}

At least $25 \%$ of mammal species are threatened with extinction, but this number is almost surely higher: $18 \%$ are data deficient and thus of unknown vulnerability (Schipper et al., 2008). The need to increase efforts and develop innovative methods for determining vertebrate biodiversity is accordingly more important than ever. The paucity of data, even for well-surveyed groups of vertebrates, relates in part to biases and inefficiencies of traditional survey techniques for uncovering cryptic, rare, and secretive species (Schnell et al., 2012, 2015; Weiskopf et al., 2018). In contrast, sequencing iDNA from blood-sucking, feces-ingesting, and carrion-feeding invertebrates' stomach contents provides an efficient method for surveying vertebrate biodiversity (Brower \& Judd, 2008; Calvignac-Spencer et al., 2013; Ngo \& Kramer, 2003; Schnell et al., 2012, 2015; Townzen, 2008).

Terrestrial leeches (Haemadipsidae) appear to be particularly effective for conservation surveys of a variety of mammals (Schnell et al., 2012, 2015). Across their broad Indo-Pacific range, haemadipsids often are abundant, appear to prey on a wide variety of vertebrates, retain bloodmeal iDNA for long periods of time, and are easy to collect as they seek out humans (Borda \& Siddall, 2010; Haeckel, 1883; Schnell et al., 2012). Schnell et al. (2012) identified six mammal species from 25 assayed leeches in Vietnam, including species that are cryptic, recently described, and likely threatened, as well as one previously unknown to the sampling area. Following up on this foundational work, Weiskopf et al. (2018) conducted a leech-based iDNA mammal survey in Bangladesh on 200 leeches, compared the results to camera trap data, and found the iDNA method to be efficient and complementary to camera traps.

Leeches assayed in Schnell et al. $(2012,2015)$ and Weiskopf et al. (2018) manifested bloodmeals from a wide range of mammalian orders (Artiodactyla, Carnivora, Lagomorpha, 
Rodentia, Scandentia, and Primates), obviously with diverse behaviors, ecologies, and body sizes. The individual leeches assessed, however, were not identified to species in Schnell et al. (2012, 2015). Weiskopf et al. (2018) did use individual iDNA determinations from single bloodmeals corresponding to known leech genotypes, and found some differences between bloodmeals from different Haemadipsa species.

Terrestrial leeches, in their own right, are a major component of tropical Asian biodiversity and are notable human and livestock pests (Borda \& Siddall, 2010). Yet few studies have been conducted on Haemadipsidae ecology, evolution, or even classification (Borda et al., 2008; Borda \& Siddall, 2010; Tan, 2008; Tessler et al., 2016). Several recent species descriptions (e.g. a cave leech from Yunnan), range extensions (into Taiwan), and nomenclatural changes (recognition of Taiwanese species) further point to poor knowledge of Asian terrestrial leeches (Lai et al. 2011, Ngamprasertwong et al. 2007, Tong et al. 2009). These aspects of leech biology are important for a more precise understanding of this abundant group and are also necessary if these leeches are to stand as valuable and accurate tools for iDNA surveys of vertebrates.

Southern Asia is an excellent place for bloodmeal survey methods, as this is the principal range of Haemadipsa, one of the two most speciose genera of terrestrial leech (Borda \& Siddall, 2010; Richardson, 1975; Sawyer, 1986; Tessler et al., 2016). More importantly, southern Asia harbors many biodiversity hotspots for mammals. For example, China, particularly Yunnan province, is an important location for conservation surveys; it is phenomenally biodiverse, with many threatened and endangered species (Yang et al., 2004). In fact, Yunnan is one of the most biodiverse areas in the world, with $50 \%$ of Chinese mammals occurring in only $4 \%$ of China's 
land area. Furthermore, Yunnan is a focal point for conservation efforts, as it contains $73 \%$ of China's priority-protected wild animal species.

This study seeks to determine the following: 1) the extent to which bloodmeals are an efficient tool for surveying and determining mammalian biodiversity in southern Asia, and 2) the genetic diversity within the leeches reviewed.

\section{Materials and methods}

Leeches were collected from a broad diversity of habitats (including farms, river edges, and forests under different levels of protection) across Yunnan and Hainan provinces in China (2014) and across Cambodia (2015). Additional leech specimens and data from Bangladesh were incorporated from Weiskopf et al. (2018). Specimens were acquired using standard techniques of walking through suitable habitat, such as humid forests and forest edges, collecting leeches from the ground or as they attached to the investigators' clothing. Leeches found feeding on investigators were not utilized for this study. Collected leeches were relaxed and fixed in either 95\% ethanol or in RNAlater (Ambion). Leech specimens were then preliminarily identified to morphogroups with stereomicroscopy.

Leech tissue was removed and further cut into pieces for proteolytic digestion and extraction; specifically, tissue was taken from anterior of the posterior sucker to the mid-body (or $2.5 \mathrm{~mm}$ maximum for larger specimens). This dissection optimized chances of finding a bloodmeal, as this is a principal area where blood is digested, while still maintaining voucher specimens with all taxonomically important characters intact. DNA was then extracted from the excised tissue using Qiagen DNeasy Blood and Tissue kits. 
The bloodmeal iDNA was amplified for vertebrate-specific 16S rRNA ( 294 bp) using $700 \mu \mathrm{M}$ of 16Scp primers (Caragiulo et al., 2014; Weiskopf et al., 2018), $0.3 \mu \mathrm{l}$ BSA, $21.3 \mu \mathrm{l}$ water, $2 \mu \mathrm{l}$ of extracted template, and GE illustra PuReTaq Ready-To-Go PCR beads. This and thermocycler profiles followed Weiskopf et al. (2018). To additionally verify each leech's identity, cytochrome $c$ oxidase subunit I (cox1) mtDNA was amplified using $500 \mu \mathrm{M}$ of $\mathrm{HHCO} 1$ (Tessler et al., n.d.) and LCO1490 primers (Folmer et al., 1994), $23.0 \mu 1$ water, $1.0 \mu 1$ of extracted template, and GE illustra PuReTaq Ready-To-Go PCR beads. Cox1 PCR protocols follow prior work on Haemadipsidae leeches (Borda et al., 2008). Amplification products were then cleaned using a 2:1 ratio of Agencourt AMPure XP (Beckman Coulter) to product. Products were cycle sequenced and ethanol precipitated prior to sequence determination via an ABI 3730 (Applied Biosystems). Sequences had primers trimmed, were reconciled, and then edited for quality in Geneious version 6.1.8 (Biomatters).

Candidate mammal hosts were identified using NCBI's blastn function based on the match with the lowest e-value. It was noted whether or not the best match was at a $1 \%$ better match in terms of percent identity. This was assumed to be the case if the first 100 matches were the same species. If several matches had identical e-values, the identity was determined only to the lowest overlapping taxonomic rank (i.e. genus or family). Chi-squared tests were conducted in R to detect differences in the success rate of BLAST matches given the preservative used, as well as preliminary differences in host specificity that the leeches in a given country may have.

Our leech cox 1 sequences were combined with cox 1 data from prior phylogenetic studies of Haemadipsidae and GenBank (Borda et al., 2008; Borda \& Siddall, 2010; Lai et al., 2011; Tessler et al., 2016; Won et al., 2014). Fully redundant sequences were then removed using the 'fastx_collapser' tool from the FASTX-Toolkit (http://hannonlab.cshl.edu/fastx_toolkit), as they 
can have negative impacts on the topological stability of maximum likelihood reconstructions and ultimately do not provide any additional information. An appropriate model was fit to the data using jModelTest 2.1.4 (Darriba et al., 2012). A maximum likelihood phylogenetic reconstruction was then conducted using the best model $(\mathrm{GTR}+\mathrm{I}+\mathrm{\Gamma})$ with the non-redundant data using Garli 2.01 (Zwickl, 2006) via the CIPRES Science Gateway (Miller et al., 2010). Support for the most likely topology was calculated using 1,000 bootstrap pseudoreplicates in the same programme and summarized on the most likely phylogenetic reconstruction with SumTrees of the DendroPy library (Sukumaran \& Holder, 2010).

\section{Results}

\section{Bloodmeal BLAST}

Approximately 750 leech specimens were examined. Vertebrate species identified from iDNA (Table 1) represent one avian order (Galliformes; found in 10 leech samples) and a large number of mammalian orders (Artiodactyla, Carnivora, Chiroptera, Primates, Rodentia, Scandentia; found in 251 leech samples). Please note that using shorter fragments of iDNA could possibly amplify a higher percentage of bloodmeals. Artiodactyl species were those most commonly amplified, representing $45 \%$ of the sequences recovered. In total, 31 species in 16 families were identified; however, identities are preliminary, as definitive identification would often require additional loci and in larger databases and is beyond the scope of this work.

The most common wild mammals found were the Asian palm civet (Paradoxurus hermaphroditus; 19 samples) and the lion-tailed macaque (Macaca silenus; 17 samples). In addition to wild animals, iDNA was identified humans and domesticated animals (Bos taurus, Bubalus bubalus, Canis lupus, Felis silvestris, Sus scrofa, and Gallus gallus). We view these 
human and domesticated animal identifications cautiously, as these taxa are sometimes lab contaminants; however, we believe our lab protocols should have mitigated such issues (see Weiskopf et al., 2018). These iDNA sequence results cannot differentiate between domesticated and wild forms of S. scrofa or G. gallus that co-occur in many of the areas reviewed.

Amplification success rates for iDNA were on average $34 \%$, but varied substantially between sites. Most notably, iDNA sequences were successfully obtained in $62 \%$ of samples from the Tarap Hill Reserve Forest site in Bangladesh, while only $11 \%$ were recovered from bloodmeals from Seima in Cambodia. The iDNA was successfully amplified whether the leech specimen was preserved in ethanol (30 amplified) or RNAlater (231 amplified; no significant difference was found, with $P=0.9991)$. Following sequence determination above, iDNA from 265 specimens were reamplified and resequenced to achieve better quality. Of these, only two resulted in amplified products that had sequences not matching what was originally determined. In both cases, each isolate contained sequences corresponding both to $P$. hermaphroditus and $B$. taurus.

The species/clade of the leech did not seem to have notable host specificity or correspond to any obvious pattern in terms of vertebrate host found. However, sample sizes precluded all but the most rudimentary comparisons. Chi-squared tests were conducted using four leech clades and three vertebrate orders in China $(P=0.4561)$ and two leech clades and three vertebrate orders in Bangladesh $(P=0.7512)$, finding no relationship between these variables. These subsets were selected as they had a minimum frequency of 10 for each leech clade and each vertebrate order. Larger sampling sizes could easily change these results.

\section{Leech relationships}


A total of 730 leech cox 1 isolates were successfully amplified and sequenced, and then combined with 104 GenBank sequences. With redundant sequences removed, there were 333 unique sequences aligned in the final matrix. Leeches evaluated phylogenetically belonged to 15 clades of Haemadipsa (Fig. 1). These clades, including H. trimaculosa and H. hainana, were generally well-supported; backbone relationships were not. Fully 107 specimens were preliminarily identified morphologically as $H$. sylvestris and yet were recovered in disparate branches of the phylogenetic reconstruction (i.e. Bangladesh Clade D and China clades F and G). At present it is unclear which of these clades represents the 'true' $H$. sylvestris, and accordingly, these clades were not named. Morphogroups generally corresponded to the 15 recovered clades. Some sister clades that appeared morphologically similar showed evidence of being genetically divergent (e.g. China clades C and D).

The most well-sampled clade, H. trimaculosa, was represented by individuals from three major regions in Cambodia. These specimens had substantial genetic diversity, which was strongly circumscribed by locality in Cambodia (Fig. 1). These regions are primarily separated by distance and by major river drainages (e.g. the Mekong River and Tonle Sap). This phylogenetic distance between geographically separate localities was reflected at other points in the reconstruction (Fig. 1). Specifically, Bangladesh clades B and C and China clades C and D were morphologically similar, sister, separated by meaningfully long branches, and geographically adjacent. Data from prior studies (e.g. Lai et al., 2011), when combined and analyzed here, sometimes showed a similar pattern for some (e.g. H. picta), but not other species (e.g. Haemadipsa rjukjuana specimens from Taiwan were not a group nor were $H$. interrupta from Thailand). Bangladesh sites harbored leech individuals from the same clade without any phylogenetic distinction between adjacent sites. Bangladesh clade A comprised leech individuals 
from all four sites. Bangladesh clades B and D had individuals from two sites and no phylogenetic separation.

\section{Discussion}

\section{Bloodmeal iDNA survey}

One of the most important findings of this bloodmeal iDNA survey (Table 1) is corroboration that leeches feed on small mammals, such as rodents, that are often missed by other generalized non-invasive methods commonly employed for mammal surveys. Camera traps are much less likely to record small mammals compared to larger ones, as they often are not sensitive enough to be triggered by small ground-dwelling species (Tobler et al., 2008). This advantage of the iDNA method over the traditional camera trap method already has been shown directly in Bangladesh by Weiskopf et al. (2018). This study expands on that finding, showing the ability of iDNA surveys to find small mammals across a number of Asian tropical ecosystems.

Similarly, our broad survey combined with prior geographically constrained studies (Schnell et al., 2012; Weiskopf et al., 2018) now makes it clear that species of Haemadipsa feed on a large variety of mammalian orders (Artiodactyla, Carnivora, Chiroptera, Lagomorpha, Rodentia, Scandentia, and Primates) at least somewhat indiscriminately across their range; something of importance to the suitability of the iDNA method. The robustness of the iDNA method is further strengthened by the fact that there are so few orders of wild mammals potentially present for which iDNA was not recovered (e.g. Pholidota [pangolins] and Proboscidea [elephants]). Pangolins, while potentially present, are rapidly decreasing across much of their ranges (Trageser et al., 2017). Elephants were known from some localities in 
Cambodia and were found using camera traps in Bangladesh (Weiskopf et al., 2018), but are
infrequent at best.

We recovered bloodmeals from three avian genera (Coturnix, Gallus, and Lophura) in the Galliformes. At least one species of these ground-dwelling birds were recovered in leech bloodmeals from each country surveyed, indicating that Haemadipsa species may regularly target such birds. Members of the Haemadipsidae have only rarely been found on birds (Bhatai \& Bora, 1973; Sawyer, 1986). All other records are largely isolated and more anecdotal. Haemadipsa leeches were discovered in one study on legs of wild pheasants (Bhatai \& Bora, 1973). Chtonobdella limbata has been found on the leg of a juvenile yellow robin and $C$. bilineata on the head of a black-backed magpie (Richardson, 1975). In Papua New Guinea and New Britain, C. novabritanniae was found in the nasal chambers of cassowaries (Richardson, 1975). Our observation that leeches in this study fed on galliform birds and not passerines may contribute to a lack of long distance dispersal for the leech.

One sample from Cambodia had a bloodmeal from a genus of leaf-nosed bat (Hipposideros). Species of terrestrial leech previously known to feed on bats are specialized cave-dwelling species: H. cavatuses from China (Yang et al., 2009) and C. jawarerensis from Papua New Guinea (Ewers, 1974; Richardson, 1974). Prior to our record, generalist Haemadipsa species have not been recorded from bats.

Many of the samples were found to have fed on domesticated animals, which is only to be expected given the mixed-use nature of southern Asian forests. Yet, notwithstanding the number of large domestic bovids (e.g. cows and domestic water buffalo), we were readily able to distinguish a gaur (Bos gaurus) isolate. This species, also known as the Indian bison, is listed by 
the International Union for Conservation of Nature and Natural Resources (IUCN) as a vulnerable species on its Red List.

While the leeches generally appear to prefer ground-dwelling species, no pattern was evident between leeches of a given clade and their prey. This lack of clade-specific host specificity and relative similarity in efficiencies between different species suggests that the iDNA technique may be robust for finding vertebrates in an area regardless of which leech species is reviewed. However, future work should strive for larger samples sizes that could rigorously test for differences in host specificity. Furthermore, this alone does not indicate the site fidelity of a given leech species, which is equally important for the iDNA method and best determined by the phylogenetic relationships between leech individuals of a given species.

\section{Leech relationships}

Broad-scale site fidelity is perhaps best exemplified in H. trimaculosa individuals from sites in Cambodia (Fig. 1). Schnell et al. (2015) have proposed that site-occupancy modelling can be applied to iDNA surveys, which corrects host species distribution maps for the ubiquitous problem of false negatives: i.e. when a host species is present in a site but not detected by the census method. Site-occupancy models achieve this correction via multiple revisits to the same site, so that detection probabilities can be estimated for each species. This is costly fieldwork, but since haematophagous leeches are abundant, Schnell et al. (2015) have suggested that subsamples of leeches from the same site could be used instead of multiple revisits. However, an important assumption of site-occupancy models is 'population closure,' which in this context amounts to the assumption that where a leech is collected is close to where the leech fell of its previous host. This assumption seems reasonable, given that an engorged leech is unlikely to travel far, but evidence so far is lacking. The correspondence between $H$. trimaculosa (as well as 
Bangladesh clades B and C and China clades C and D) and geographic boundaries does not provide evidence for population closure, but does leave open the possibility that further examination may find this assumption to be met.

Several other clades do not show these patterns. Bangladesh clade A shows little phylogenetic patterning within the clade and yet is distributed across four distinct localities in Bangladesh. This points to the possibility that leeches in some clades are more mobile than others and less accurate regarding the exact location of a leech's host. However, this finding is preliminary and could be due to other factors. For example, the leeches in this clade, while somewhat geographically disjunct $(\sim 30 \mathrm{~km})$, may have contiguous population structure throughout forested areas that connect in India.

In addition to the matter of site fidelity, previously, it had been argued that Haemadipsa leeches are unlikely to have iDNA from more than one host (Schnell et al., 2012). Our Sanger sequencing protocol finds that individual leeches do largely retain a single bloodmeal. Only two of our surveyed leeches had conflicting nucleotide peaks that when reamplified and resequenced (over 200 samples) for improved quality had BLAST matches to two separate species. However, this is a rudimentary way to distinguish multiple hosts and surely misses additional cases of mixed bloodmeals.

Haemadipsa trimaculosa was the species most frequently encountered in this study and is the first records of this species (along with H. interrupta) in Cambodia. No prior Haemadipsa records exist this country. The only other records for H. trimaculosa are from eastern (mostly adjacent) parts of Thailand, where the species was first described (Ngamprasertwong et al., 2007), and from adjacent and close provinces of Laos (Vongsombath et al., 2011). 
Given what is known of the range of H. trimaculosa, the sampling presented here appears to be the most thorough molecular sampling and evaluation ever conducted for any leech species, except perhaps Hirudo medicinalis. Few species of leech have been molecularly compared using more than two or three samples, and none of these studies focused on the terrestrial leeches that are most useful for iDNA surveys. Hirudo medicinalis and Hirudo verbana have been molecularly compared in a more thorough fashion than other leeches, with sampling occurring over large portions of their range; however, at most around 60 wild caught specimens per species are used in a given study (Siddall et al., 2007; Trontelj \& Utevsky, 2012; Utevsky \& Trontelj, 2015). With so few studies looking at the genetic structuring of leeches across any significant geographic range, the geographically bound, genetically defined clades we found for $H$. trimaculosa highlight just how little is known about leech diversity and biology.

Finally, $H$. sylvestris and H. montana appear to be polyphyletic and in clear need of revision. This was also the case for many of our unnamed clades. Substantial further revisionary work is needed on the group, but requires additional collections from type localities, especially for those species originally described from India.

\section{Acknowledgements}

Thanks to Mohammad Faiz and George Amato for help with molecular work; Bob Murphy, Li Zongxu, Wu Chunying, Chen YiZhang, Yang Yahan, Nina You, the Wildlife Conservation Society, and Flora and Fauna International for aid in fieldwork; and the U. of Delaware College of Agriculture and Natural Resources for financially supporting the collection and analysis of the Bangladesh samples. We especially thank NSF-EAPSI for a fellowship funding MT (\#1414639), NSF-PEET funding MES (\#0119329), and the Niarchos Foundation for funding to MES. 


\section{References}

Bhatai, M. L., \& Bora, S. S. (1973). Bionomics and distribution of the land leeches of Kumaon Hills, U.P. Journal of the Bombay Natural History Society, 70, 36-56.

Borda, E., Oceguera-Figueroa, A., \& Siddall, M. E. (2008). On the classification, evolution and biogeography of terrestrial haemadipsoid leeches (Hirudinida: Arhynchobdellida: Hirudiniformes). Molecular Phylogenetics and Evolution, 46, 142-154.

Borda, E., \& Siddall, M. E. (2010). Insights into the evolutionary history of Indo-Pacific bloodfeeding terrestrial leeches (Hirudinida: Arhynchobdellida: Haemadipsidae). Invertebrate Systematics, 24, 456.

Calvignac-Spencer, S., Merkel, K., Kutzner, N., Kühl, H., Boesch, C., Kappeler, P. M., ... Leendertz, F. H. (2013). Carrion fly-derived DNA as a tool for comprehensive and costeffective assessment of mammalian biodiversity. Molecular Ecology, 22, 915-924.

Caragiulo, A., Dias-Freedman, I., Clark, J. A., Rabinowitz, S., \& Amato, G. (2014).

Mitochondrial DNA sequence variation and phylogeography of Neotropic pumas (Puma concolor). Mitochondrial DNA, 25, 304-312.

Darriba, D., Taboada, G. L., Doallo, R., \& Posada, D. (2012). jModelTest 2: more models, new heuristics and parallel computing. Nature Methods, 9, 772.

Ewers, W. H. (1974). Trypanosoma aunawa sp. n. from an insectivorous bat, Miniopterus tristris, in New Guinea, which may be transmitted by a leech. Journal of Parasitology, 60, $172-178$.

Folmer, O., Black, M., Hoeh, W., Lutz, R., \& Vrijenhoek, R. (1994). DNA primers for amplification of mitochondrial cytochrome c oxidase subunit I from diverse metazoan 
invertebrates. Molecular Marine Biology and Biotechnology, 3, 294-299.

Haeckel, E. (1883). A Visit to Ceylon. London, UK: Kegan Paul, Trench \& Co.

Lai, Y.-T., Nakano, T., \& Chen, J.-H. (2011). Three species of land leeches from Taiwan, Haemadipsa rjukjuana comb. n., a new record for Haemadipsa picta Moore, and an updated description of Tritetrabdella taiwana (Oka). ZooKeys, 139, 1-22.

Miller, M. A., Pfeiffer, W., \& Schwartz, T. (2010). Creating the CIPRES Science Gateway for inference of large phylogenetic trees. In 2010 Gateway Computing Environments Workshop. New Orleans, USA: IEEE Computer Society.

Ngamprasertwong, T., Thirakhupt, K., \& Panha, S. (2007). Two new species of land leeches from Thailand (Hirudiniformes: Haemadipsidae). The Natural History Journal of Chulalongkorn University, 7, 155-159.

Ngo, K. A., \& Kramer, L. D. (2003). Identification of mosquito bloodmeals using polymerase chain reaction (PCR) with order-specific primers. Journal of Medical Entomology, 40, 215222.

Richardson, L. R. (1974). A new troglobitic quadrannulate land-leech from Papua (Hirudinoidea: Haemadipsidae s. 1.). Proceedings of the Linnean Society of New South Wales. Linnean Society of New South Wales, 99, 57-68.

Richardson, L. R. (1975). A contribution to the general zoology of the land-leeches (Hirudinea: Haemadipsoidea superfam. nov.). Acta Zoologica Academiae Scientiarum Hungaricae , 21, $119-152$.

Sawyer, R. T. (1986). Leech biology and behaviour: feeding biology, ecology, and systematics. Oxford, UK: Clarendon Press.

Schipper, J., Chanson, J. S., Chiozza, F., Cox, N. A., Hoffmann, M., Katariya, V., .. Young, B. 
Schnell, I. B., Sollmann, R., Calvignac-Spencer, S., Siddall, M. E., Yu, D. W., Wilting, A., \& Gilbert, M. T. P. (2015). iDNA from terrestrial haematophagous leeches as a wildlife surveying and monitoring tool - prospects, pitfalls and avenues to be developed. Frontiers in Zoology, 12, 24.

Schnell, I. B., Thomsen, P. F., Wilkinson, N., Rasmussen, M., Jensen, L. R. D., Willerslev, E., ... Gilbert, M. T. P. (2012). Screening mammal biodiversity using DNA from leeches. Current Biology: CB, 22, R262-3.

Siddall, M. E., Trontelj, P., Utevsky, S. Y., Nkamany, M., \& Macdonald, K. S. (2007). Diverse molecular data demonstrate that commercially available medicinal leeches are not Hirudo medicinalis. Proceedings of the Royal Society B: Biological Sciences, 274, 1481-1487.

Sukumaran, J., \& Holder, M. T. (2010). DendroPy: a Python library for phylogenetic computing. Bioinformatics , 26, 1569-1571.

Tan, E. (2008). Progress in the study of ecology, zoogeography, group, control repellent and medical usage of Hirudinea in China. Acta Ecologica Sinica, 28, 6272-6281.

Tessler, M., Barrio, A., Borda, E., Rood-Goldman, R., Hill, M., \& Siddall, M. E. (2016). Description of a soft-bodied invertebrate with microcomputed tomography and revision of the genus Chtonobdella (Hirudinea: Haemadipsidae). Zoologica Scripta, 45, 552-565.

Tessler, M., Siddall, M. E., \& Oceguera-Figueroa, A. Leeches from Chiapas, Mexico with a new species of Erpobdella (Hirudinida: Erpobdellidae). American Museum Novitates, in press.

Tobler, M. W., Carrillo-Percastegui, S. E., Leite Pitman, R., Mares, R., \& Powell, G. (2008). An evaluation of camera traps for inventorying large- and medium-sized terrestrial rainforest 
mammals. Animal Conservation, 11, 169-178.

Townzen, J. S., Brower, A. V. Z., \& Judd, D. D. (2008). Identification of mosquito bloodmeals using mitochondrial cytochrome oxidase subunit I and cytochrome b gene sequences. Medical and Veterinary Entomology, 22, 386-393.

Trageser, S. J., Ghose, A., Faisal, M., Mro, P., Mro, P., \& Rahman, S. C. (2017). Pangolin distribution and conservation status in Bangladesh. PloS One, 12, e0175450.

Trontelj, P., \& Utevsky, S. Y. (2012). Phylogeny and phylogeography of medicinal leeches (genus Hirudo): fast dispersal and shallow genetic structure. Molecular Phylogenetics and Evolution, 63, 475-485.

Utevsky, S., \& Trontelj, P. (2015). Phylogeography of the southern medicinal leech, Hirudo verbana: a response to Živić et al. (2015). Aquatic Ecology, 50, 97-100.

Vongsombath, C., de Boer, H. J., \& Pålsson, K. (2011). Keeping leeches at bay: field evaluation of plant-derived extracts against terrestrial blood-sucking leeches (Haemadipsidae) in Lao PDR. Acta Tropica, 119, 178-182.

Weiskopf, S. R., McCarthy, K. P., Tessler, M., Rahman, H. A., McCarthy, J. L., Hersch, R., Faisal, M. M., \& Siddall, M. E. (2018). Using terrestrial haematophagous leeches to enhance tropical biodiversity monitoring programs in Bangladesh. Journal of Applied Ecology, in press.

Won, S., Park, B. K., Kim, B. J., Kim, H. W., Kang, J. G., Park, T. S., ... Chae, J. S. (2014). Molecular identification of Haemadipsa rjukjuana (Hirudiniformes: Haemadipsidae) in Gageo Island, Korea. The Korean Journal of Parasitology, 52, 169-175.

Yang, T., Mo, X., \& Wang, D. (2009). A new species of cavernous blood sucking land leech (Hirudinea, Haemadipsidae) in the west of Yunnan Province, China. Acta Zootaxonomica 
Sinica/Dongwu Fenlei Xuebao, 34, 125-129.

Yang, Y., Tian, K., Hao, J., Pei, S., \& Yang, Y. (2004). Biodiversity and biodiversity conservation in Yunnan, China. Biodiversity and Conservation, 13, 813-826.

Zwickl, D. J. (2006). Genetic algorithm approaches for the phylogenetic analysis of large

biological sequence datasets under the maximum likelihood criterion. Austin, USA: The University of Texas at Austin. 


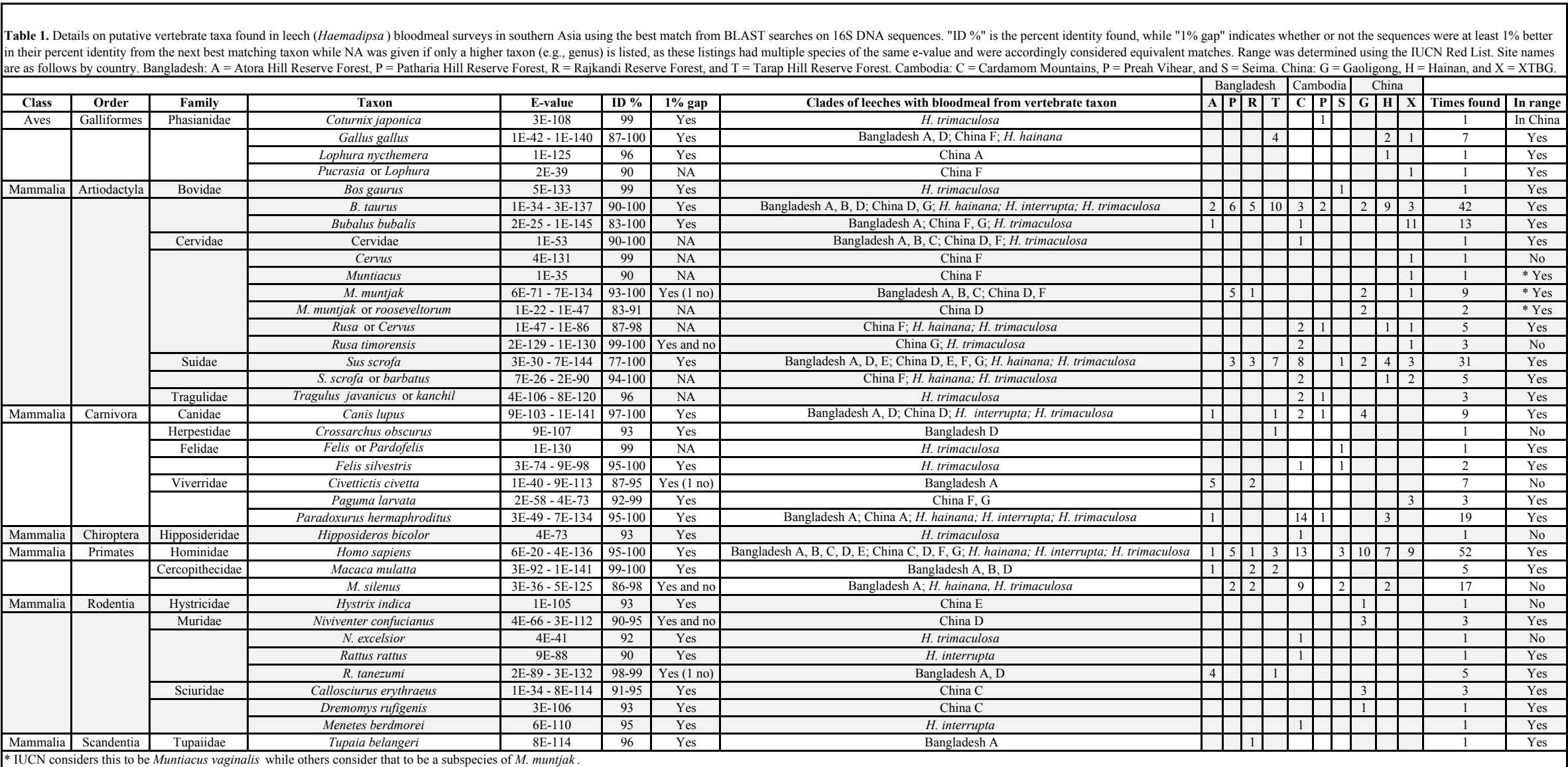


Fig. 1. The most likely phylogenetic reconstruction of Haemadipsa leeches, with sequences new to this study bolded and underlined. Collection localities are covered by black ovals for the rough range in a given site, with a different colour for each symbol indicating the specific site. The shape of each symbol within these ovals represents the country of a given site, with circles for Bangladesh and triangles for China. These symbols are also displayed next to the clades that had samples from these localities. Bootstrap support values above $50 \%$ are displayed as a measure of support. 